\title{
LARGE TIME BEHAVIOR FOR A NONLOCAL DIFFUSION EQUATION WITH ABSORPTION AND BOUNDED INITIAL DATA: THE SUBCRITICAL CASE
}

\author{
ARIEL SALORT, JOANA TERRA, AND NOEMI WOLANSKI
}

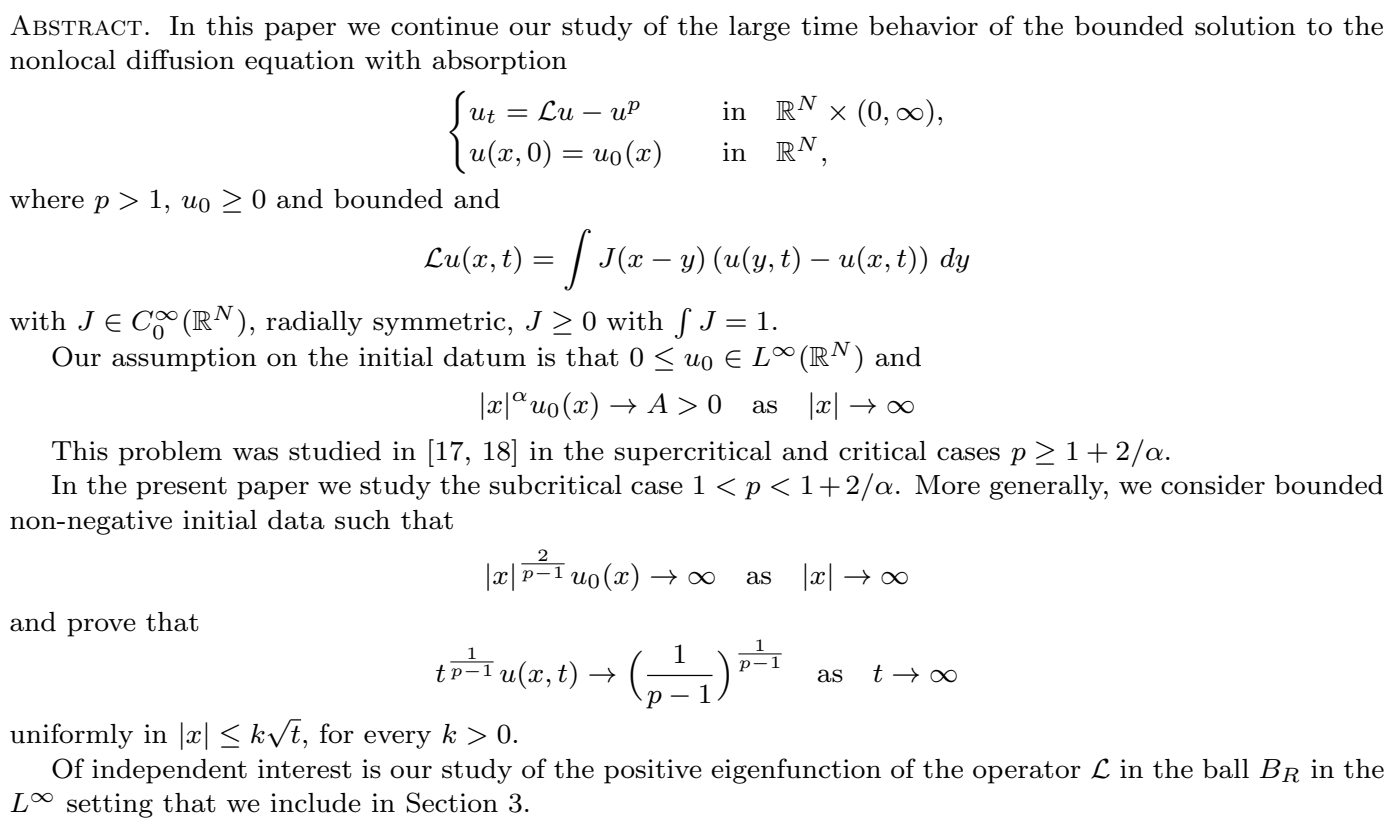
$L^{\infty}$ setting that we include in Section 3 .

\section{INTRODUCTION}

In this paper we continue our study of the large time behavior of the solution to the nonlocal diffusion equation with absorption

$$
\begin{cases}u_{t}=\mathcal{L} u-u^{p} & \text { in } \mathbb{R}^{N} \times(0, \infty) \\ u(x, 0)=u_{0}(x) & \text { in } \quad \mathbb{R}^{N}\end{cases}
$$

where $p>1, u_{0} \geq 0$ and bounded and

$$
\mathcal{L} u(x, t)=\int J(x-y)(u(y, t)-u(x, t)) d y
$$

with $J \in C_{0}^{\infty}\left(\mathbb{R}^{N}\right)$, radially symmetric, $J \geq 0$ with $\int J=1$.

Our assumption on the initial datum is that $0 \leq u_{0} \in L^{\infty}\left(\mathbb{R}^{N}\right)$ and

$$
|x|^{\alpha} u_{0}(x) \rightarrow A>0 \quad \text { as } \quad|x| \rightarrow \infty
$$

These kind of nonlocal diffusions appear in several applications such as population dynamics, disease propagation, image enhancement, etc (see, for instance, $[2,3,4,5,6,9,12,19]$.

2010 Mathematics Subject Classification. 35R09, 45K05, 35K57 35 B40 .

Key words and phrases. Nonlocal diffusion, Large time behavior.

Supported by CONICET PIP625 Res. 960/12, ANPCyT PICT-2012-0153 and UBACYT X117. 
When the kernel $J$ in the nonlocal operator (1.2) satisfies the hypotheses in this paper, the long time behavior of the solutions is closely related to that of the corresponding problem for the heat operator with a diffusivity related to the kernel $J$ (see, for instance, $[7,10,14,16,17,18]$ ).

In [16] the authors started the study of (1.1) when $u_{0} \in L^{1}\left(\mathbb{R}^{N}\right)$, in the supercritical case $p>1+2 / N$. Then, in $[17,18]$ we studied this problem under assumption $(1.3)$.

The main question we address is what is the interplay between the parameters $p, \alpha$ and the dimension $N$ in the large time behavior of the solution.

In $[17,18]$ the critical and supercritical cases were studied. This is, we assumed that, either $u_{0} \in L^{1}\left(\mathbb{R}^{N}\right)$ and $p \geq 1+2 / N$ (completing the results of [16] by considering the critical case), or $0<\alpha<N$ and $p \geq 1+2 / \alpha$. Also some intermediate asymptotics for $u_{0}$ involving logarithms where considered in [18], always in the supercritical case.

In the present paper we complete our study by considering the subcritical case $1<p<1+2 / \alpha$ that was left open in the previous articles.

The critical value $p_{c}=1+2 / \alpha$ is the one that makes diffusion and absorption of the "same size". It is interesting to observe that this critical value depends on the size of the initial condition at infinity.

In the supercritical case, diffusion wins and the reaction component disappears in the long run. In the critical case, both diffusion and reaction remain in the time asymptotics (see $[16,17,18]$ ).

In the present paper we show that, in the subcritical case, only reaction remains in the large time behavior and the solution behaves as that of the equation

$$
u_{t}=-u^{p}, \quad u(1)=\left(\frac{1}{p-1}\right)^{\frac{1}{p-1}} .
$$

This is

$$
t^{\frac{1}{p-1}} u(x, t) \rightarrow\left(\frac{1}{p-1}\right)^{\frac{1}{p-1}} \text { as } t \rightarrow \infty \quad \text { uniformly in }\{|x| \leq k \sqrt{t}\}
$$

for every $k>0$.

It is interesting to observe that the final profile is independent of the initial datum $u_{0}$ as long as it is bounded and satisfies (1.3). In the critical and supercritical cases, both the constant $A$ and the exponent $\alpha$ in (1.3) intervene in the time asymptotics.

Our result is similar to the one obtained by Gmira and Veron in [13] for the heat equation with absorption. As in [13], we get this behavior for any nonnegative and bounded initial datum $u_{0}$ such that

$$
|x|^{\frac{2}{p-1}} u_{0}(x) \rightarrow \infty \quad \text { as } \quad|x| \rightarrow \infty
$$

thus allowing a more general behavior of $u_{0}$ at infinity than the one in (1.3).

In this paper we follow the ideas of [13] where the authors constructed subsolutions of separated variables with the right asymptotic behavior. These subsolutions involve the positive eigenfunctions $h_{R}$ of the laplacian in the balls $B_{R}$, normalized so that the $\left\|h_{R}\right\|_{L^{\infty}\left(B_{R}\right)}=1$.

The authors make use of the scaling invariance of the laplacian so that $h_{R}(x)=h_{1}(x / R)$ and the principal eigenvalue $\lambda_{R}=R^{-2} \lambda_{1}$.

One of the main differences when dealing with problem (1.1) is the lack of any scaling invariance of the problem. Nevertheless, a parabolic scaling leads -in the limit of the scaling parameter going to infinity- to the heat equation with diffusivity $A(J)=\frac{1}{2 N} \int J(z)|z|^{2} d z$. And this fact explains, in a way, the interplay between the time asymptotics of the nonlocal diffusion equation and that of the heat equation with diffusivity $A(J)$, as was made clear in [18].

This scaling property was also the basis for the understanding of the behavior as $|x| \rightarrow \infty$ of the solution to

$$
\left\{\begin{array}{lll}
\mathcal{L} \phi=0 & \text { in } & \mathbb{R}^{N} \backslash \Omega \\
\phi=1 & \text { in } & \Omega \\
\phi(x) \rightarrow 0 & \text { as } & |x| \rightarrow \infty .
\end{array}\right.
$$

with $\Omega$ an open bounded set, studied in [8]. 
One of the main contributions of the present paper is a thorough study of the positive eigenfunction $H_{R}$ to the nonlocal operator $\mathcal{L}$ in the ball $B_{R}$ with Dirichlet boundary conditions $H_{R}=0$ in $\mathbb{R}^{N} \backslash B_{R}$, normalized so that $\left\|H_{R}\right\|_{L^{\infty}}=1$.

This study was initiated in [11] where the existence of a principal eigenvalue $\Lambda_{R}$ associated to a positive eigenfunction was proved. Moreover, in [11] the authors proved that, asymptotically the principal eigenvalue behaves as that of the laplacian with diffusivity $A(J)$. This is,

$$
R^{2} \Lambda_{R} \rightarrow A(J) \lambda_{1} \quad \text { as } \quad R \rightarrow \infty .
$$

In [11] the authors also studied the associated eigenfunction in the $L^{2}$ setting and they proved that, after rescaling to the unit ball with an $L^{2}$ normalization, one gets convergence in $L^{2}$ to the positive eigenfunction of the laplacian in the unit ball with Dirichlet boundary conditions and unit $L^{2}-$ norm.

In the present paper, due to the application to the study of the asymptotics of (1.1) we have in mind, we are interested in a different normalization and convergence. Namely, we normalize so that the $L^{\infty}-$ norm is preserved and prove uniform convergence in the unit ball.

In order to get this kind of compactness, the arguments in [11] cannot be applied. Instead, we get uniform bounds for the derivatives of the rescaled eigenfunctions $\widetilde{H}_{R}(x)=H_{R}(R x)$, on smaller balls $B_{r}$ with $0<r<1$, by using an integral representation formula for $H_{R}$ and a precise decay in terms of $R$ of $H_{R}$ in a neighborhood of the boundary of $B_{R}$. To this end, we construct an upper barrier. This barrier also allows to get uniform smallness of the rescaled eigenfunctions and their limits in a neighborhood of $\partial B_{1}$ that gives, in particular, uniform convergence in the whole ball. The uniform limit is then identified as being $h_{1}$, the positive eigenfunction of the laplacian in the unit ball with Dirichlet boundary conditions and unit $L^{\infty}-$ norm.

We believe that the results concerning the eigenfunctions $H_{R}$ are of independent interest.

The paper is organized as follows. In Section 2 we state the results of [11] on the principal eigenvalue of the operator $\mathcal{L}$ with Dirichlet boundary conditions set in the ball $B_{R}$. Then, in Section 3 we perform our study of the eigenfunctions associated to the principal eigenvalues in the $L^{\infty}$ setting. In Section 4 we construct a subsolution to (1.1) by following the ideas of [13] for the heat equation. Due to the lack of any regularizing effect of the nonlocal operator, we need to prove that $\inf _{B_{R}} u(\cdot, t)>0$ for every $R>0, t>0$ (Lemma 4.1). Finally, in Section 5 we prove our main result, namely that (1.4) is satisfied.

\section{Definitions and Preliminary Results}

In this section we discuss notation and basic definitions. Moreover we state some previous results on the first eigenvalue of the nonlocal problem with Dirichlet boundary conditions in a ball.

Let $R>0$ and define the ball of radius $R$ as

$$
B_{R}=\left\{x \in \mathbb{R}^{n}:|x|<R\right\}
$$

We denote by $\lambda_{R}$ the first eigenvalue of the laplacian in $B_{R}$. That is, $\lambda_{R}$ verifies that there is a solution to the following problem,

$$
\begin{cases}-\Delta u=\lambda_{R} u & \text { in } B_{R}, \\ u=0 & \text { on } \partial B_{R}, \\ u>0 & \text { in } B_{R} .\end{cases}
$$

We know that $\lambda_{R}$ is simple. Let us call $h_{R}$ the associated eigenfunction satisfying

$$
0<h_{R}(x) \leq 1=\max _{x \in B_{R}} h_{R}(x) \quad \text { in } B_{R} .
$$

It is well known that, due to the scaling of the laplacian there holds that, $\lambda_{R}=R^{-2} \lambda_{1}$. 
We now consider the nonlocal eigenvalue problem,

$$
\left\{\begin{array}{cl}
-\mathcal{L} u(x)=\Lambda_{R} u(x) & \text { in } B_{R} \\
u=0 & \text { in } \mathbb{R}^{N} \backslash B_{R}, \\
u>0 & \text { in } B_{R} .
\end{array}\right.
$$

where $\mathcal{L} u(x)=\int J(x-y)(u(y, t)-u(x, t)) d y$ and $J \in C_{0}^{\infty}\left(\mathbb{R}^{N}\right)$ is radially symmetric and $J \geq 0$ with $\int J=1$.

It was proved in [11] that such an eigenvalue exists, it is simple and moreover,

$$
\Lambda_{R} \sim A(J) \frac{\lambda_{1}}{R^{2}} \quad \text { as } R \rightarrow+\infty
$$

with

$$
A(J)=\frac{1}{2 N} \int_{\mathbb{R}^{N}} J(z)|z|^{2} d z
$$

This is,

$$
\Lambda_{R}=A(J)(1+o(1)) \frac{\lambda_{1}}{R^{2}} \quad \text { as } R \rightarrow+\infty .
$$

Consequently, the first eigenvalue $\Lambda_{R}$ for the nonlocal problem (2.3) behaves asymptotically as the first eigenvalue $\lambda_{R}$ of the laplacian (2.1), as $R$ tends to infinity.

Moreover, in [11] the authors proved that $\Lambda_{R}$ is given variationally as

$$
\Lambda_{R}=\inf _{\substack{0 \neq u \in L^{2}\left(B_{R}\right) \\ u=0 \text { in } B_{R}^{c}}} \frac{1}{2} \frac{\int J(x-y)(u(x)-u(y))^{2} d x d y}{\int u^{2}(x) d x} .
$$

\section{Some RESUlts On the EIGENFUnCtions}

In this section we study the eigenfunctions of the nonlocal problem in the ball $B_{R}$.

The eigenfunction problem was studied in [11] in the $L^{2}$ setting. This is, in [11] the authors consider the family of eigenfunctions normalized as to have the $L^{2}\left(B_{R}\right)$-norm equal to 1 and prove that, when properly rescaled, they converge to the unique positive eigenfunction of the laplacian in the unit ball with $L^{2}\left(B_{1}\right)$-norm equal to 1 .

In the present paper we are interested in the family $H_{R}$ of positive eigenfunctions normalized so that the $L^{\infty}\left(B_{R}\right)$-norm is 1 . We prove that, when properly rescaled, they converge to the unique positive eigenfunction of the laplacian in the unit ball with the same normalization. The convergence is uniform in the unit ball.

In order to get our result, we cannot use the compactness argument of [11] that holds only in $L^{p}$ for $p<\infty$. Instead, we use Arzelà-Ascoli. To this end, we get uniform estimates of the derivatives of the rescaled eigenfunctions on compact subsets of the unit ball. The argument is delicate and uses a precise decay, in terms of $R$, of $H_{R}$ in a neighborhood of the boundary of $B_{R}$. This decay is obtained by comparison with a supersolution that we construct to this end. In this way we obtain uniform convergence on compact subsets of the unit ball.

The supersolution also allows us to prove that the rescaled eigenfunctions $\widetilde{H}_{R}$ are smaller than any positive constant in a neighborhood of $\partial B_{1}$ if $R$ is large. This, in turn, gives that the convergence is uniform in the unit ball to a function that is continuous in the closure and vanishes on the boundary. This limit function is therefore $h_{1}$.

In this way, we get our main result in this section. Namely,

Theorem 3.1. Let $\mathcal{L}$ the operator in (1.2). Let $\Lambda_{R} \in \mathbb{R}, H_{R} \in C\left(\bar{B}_{R}\right), H_{R}=0$ in $\mathbb{R}^{N} \backslash B_{R}$, be the unique solution to

$$
\begin{cases}-\mathcal{L} H(x)=\Lambda_{R} H(x) & \text { in } \quad B_{R}, \\ H(x)=0 & \text { in } \mathbb{R}^{N} \backslash B_{R}, \\ H(x)>0 & \text { in } \quad B_{R}\end{cases}
$$


obtained in [11], with the normalization $0<H(x) \leq 1=\left\|H_{R}\right\|_{L^{\infty}\left(B_{R}\right)}$ for $x \in B_{R}$.

Let $\widetilde{H}_{R}(x)=H_{R}(R x)$ for $x \in B_{1}$ and $h_{1} \in C^{\infty}\left(B_{1}\right) \cap C\left(\bar{B}_{1}\right)$ the positive eigenfunction of the laplacian in the unit ball such that $\left\|h_{1}\right\|_{L^{\infty}\left(B_{1}\right)}=1$. Then,

$$
\widetilde{H}_{R} \rightarrow h_{1} \quad(R \rightarrow \infty) \text { uniformly in } B_{1} .
$$

For the proof of this theorem we need a couple of lemmas.

Lemma 3.2. Let $R_{n} \rightarrow \infty$ be such that $\widetilde{H}_{R_{n}} \rightarrow H$ in $L_{l o c}^{1}\left(B_{1}\right)$. Then $H$ is a solution to,

$$
\left\{\begin{array}{lll}
-\Delta H=\lambda_{1} H, & \text { in } & B_{1} \\
H=0 & \text { on } & \partial B_{1} .
\end{array}\right.
$$

Proof. In order to prove that $H$ is a weak solution to the equation, we let $\phi \in C_{0}^{\infty}\left(B_{1}\right)$ and,

$$
\begin{aligned}
& A(J) \int_{B_{1}} H(x) \Delta \phi(x) d x= \\
& =A(J) \int_{B_{1}} \widetilde{H}_{R_{n}}(x) \Delta \phi(x) d x+A(J) \int_{B_{1}}\left(H(x)-\widetilde{H}_{R_{n}}(x)\right) \Delta \phi(x) d x \\
& =\int_{B_{1}} \widetilde{H}_{R_{n}}(x) R^{2}\left(J_{R} * \phi(x)-\phi(x)\right) d x+A(J) \int_{B_{1}}\left(H(x)-\widetilde{H}_{R_{n}}(x)\right) \Delta \phi(x) d x \\
& \quad-\int_{B_{1}} \widetilde{H}_{R_{n}}(x)\left(R^{2}\left(J_{R} * \phi(x)-\phi(x)\right)-A(J) \Delta \phi(x)\right) d x \\
& \quad \int_{B_{1}}\left(J_{R} * \widetilde{H}_{R_{n}}(x)-\widetilde{H}_{R_{n}}(x)\right) \phi(x) d x \\
& \quad+A(J) \int_{B_{1}}\left(H(x)-\widetilde{H}_{R_{n}}(x)\right) \Delta \phi(x) d x-\int_{B_{1}} \widetilde{H}_{R_{n}}(x) O\left(R^{-3}\right) d x \\
& =-R^{2} \Lambda_{R} \int_{B_{1}} \widetilde{H}_{R_{n}}(x) \phi(x) d x+A(J) \int_{B_{1}}\left(H(x)-\widetilde{H}_{R_{n}}(x)\right) \Delta \phi(x) d x \\
& \quad-\int_{B_{1}} \widetilde{H}_{R_{n}}(x) O\left(R^{-3}\right) d x .
\end{aligned}
$$

Since $\widetilde{H}_{R_{n}} \rightarrow H$ strongly in $L^{2}\left(B_{1}\right)$ and $R^{2} \Lambda_{R} \rightarrow A(J) \lambda_{1}$, by taking limit as $n$ tends to infinity we obtain,

$$
A(J) \int_{B_{1}} H(x) \Delta \phi(x) d x=-\lambda_{1} A(J) \int_{B_{1}} H(x) \phi(x) d x,
$$

that is, $H$ satisfies the equation $-\Delta H=\lambda_{1} H$, in $B_{1}$.

Our next result is the construction of a barrier for $H_{R}$.

Lemma 3.3. Let $h_{1}$ be the positive eigenfunction corresponding to the first eigenvalue $\lambda_{1}$ of the laplacian in $B_{1}$ with Dirichlet boundary conditions and the normalization $1=\max _{x \in B_{1}} h_{1}(x)$. Let us consider the function

$$
v(x)=h_{1}\left(\frac{x}{2 R}\right) \quad \text { for } \quad x \in B_{2 R} .
$$

There exists $C>0, R_{0}>0$ such that

$$
C \mathcal{L} v(x) \leq \mathcal{L} H_{R} \quad \text { in } \quad B_{R} \quad \text { if } \quad R \geq R_{0} .
$$

Proof. Assume $R \geq 1$. By using Taylor's expansion and the symmetry of $J$ we get for $x \in B_{R}$,

$$
\mathcal{L} v(x)=A(J) \Delta v(x)+O\left(\max _{|\beta|=4}\left\|D^{\beta} v\right\|_{L^{\infty}\left(B_{R+1}\right)}\right) .
$$


Then,

$$
\begin{aligned}
\mathcal{L} v & =\frac{A(J)}{4} R^{-2} \Delta h_{1}\left(\frac{x}{2 R}\right)+O\left(R^{-4}\right) \\
& =-\lambda_{1} \frac{A(J)}{4} R^{-2} h_{1}\left(\frac{x}{2 R}\right)+O\left(R^{-4}\right) \\
& \leq-\frac{1}{8} \lambda_{1} A(J) R^{-2} h_{1}\left(\frac{x}{2 R}\right)
\end{aligned}
$$

if $R$ is large.

Here we have used that there exists a positive constant $c$ such that,

$$
c<h_{1}\left(\frac{x}{2 R}\right) \quad x \in B_{R} .
$$

Finally, since $\lambda_{1} A(J) R^{-2}=\Lambda_{R}+o(1)$, we get for $R$ large enough,

since $0 \leq H_{R} \leq 1$.

$$
\mathcal{L} v \leq-\frac{1}{16} \Lambda_{R} h_{1}\left(\frac{x}{2 R}\right) \leq-\frac{c}{16} \Lambda_{R} \leq-\frac{c}{16} \Lambda_{R} H_{R}=\frac{c}{16} \mathcal{L} H_{R}
$$

Recall that $h_{1}$ is radially symmetric, radially decreasing, smooth with $h_{1}(0)=1$. Let $\eta$ such that $h_{1}(x)=\eta(|x|)$.

Now we use the supersolution constructed in Lemma 3.3 in order to bound $H_{R}$. There holds,

Lemma 3.4. Let $\eta(|x|)=h_{1}(x)$ with $h_{1}$ as in Lemma 3.3. There exist constants $C, C_{0}>0$ and $R_{0}>0$ such that,

$$
H_{R}(x) \leq C\left\{\eta\left(\frac{|x|}{2 R}\right)-\eta\left(\frac{1}{2}\right)+\frac{C_{0}}{R}\right\} \quad \text { if } \quad R \geq R_{0} .
$$

Proof. In Lemma 3.3 we found a constant $C>0$ and $R_{0}>0$ such that, for any $C_{0} \in \mathbb{R}, R \geq R_{0}$, the function

satisfies

$$
w(x)=C\left\{\eta\left(\frac{|x|}{2 R}\right)-\eta\left(\frac{1}{2}\right)+\frac{C_{0}}{R}\right\}
$$

$$
\mathcal{L} w \leq \mathcal{L} H_{R} \quad \text { in } \quad B_{R} .
$$

In order to be able to apply the comparison principle we need to show that, for some constant $C_{0}$, there holds that

$$
w \geq 0 \quad \text { in } \quad\left\{x \in R^{N} \backslash B_{R} / \operatorname{dist}\left(x, B_{R}\right)<1\right\}=\{R \leq|x|<R+1\} .
$$

And, in fact (3.3) holds if $C_{0} \geq\left\|\eta^{\prime}\right\|_{L^{\infty}(0,1)}$.

Finally, by applying the Comparison Principle, the lemma is proved.

From this lemma we get the following corollary that will be used to bound the derivatives of $\widetilde{H}_{R}$.

Corollary 3.5. There exists a constant $K>0$ such that

$$
J * H_{R} \leq \frac{K}{R} \quad \text { in } \quad\{R \leq|x|<R+1\} .
$$

Proof. Let $R \leq|x|<R+1$. Then, if $J(x-y) H_{R}(y) \neq 0$, there holds that $R-1 \leq|y|<R$. Therefore,

$$
H_{R}(y) \leq w(y)=C\left\{\eta\left(\frac{|y|}{2 R}\right)-\eta\left(\frac{1}{2}\right)+\frac{C_{0}}{R}\right\} \leq \frac{K}{R}
$$

for a certain constant $K>0$ and,

$$
\left(J * H_{R}\right)(x)=\int J(x-y) H_{R}(y) d y \leq \frac{K}{R} .
$$


In order to prove our main result in this section, we will use an integral representation formula for $H_{R}$. To this end, let us recall some results on the fundamental solution to the operator $\partial_{t}-\mathcal{L}$. is

In [7] the authors found that the fundamental solution of the nonlocal operator $\partial_{t}-L$ in the whole space,

$$
F(x, t)=e^{-t} \delta(x)+\omega(x, t)
$$

where $\delta$ is the Dirac mass at the origin in $\mathbb{R}^{N}$ and $\omega$ is a smooth function.

Then, in [18] pointwise and integral estimates for $w$ and its derivatives where obtained. In particular,

$$
|\nabla \omega(x, t)| \leq C \frac{t}{|x|^{N+3}},
$$

and,

$$
\int_{\mathbb{R}^{N}}|\nabla \omega(x, t)| \leq C t^{-\frac{1}{2}}
$$

We can now prove our main result in this section.

Proof of Theorem 3.1. The proof follows from the Arzelá-Ascoli Theorem.

In order to get uniform estimates of the derivatives of $\widetilde{H}_{R}$ let us observe that the first eigenfunction of (2.3) is the unique bounded solution of the following non-homogeneous equation defined in the whole $\mathbb{R}^{N}$,

$$
\begin{cases}w_{t}-\mathcal{L} w=\Lambda_{R} w-\mathcal{X}_{B_{R}^{c}}(J * w) & \text { in } \mathbb{R}^{N} \times(0, \infty), \\ w(x, 0)=H_{R}(x) & \text { in } \mathbb{R}^{N} .\end{cases}
$$

As the solution of (3.6) is defined in the whole space, it can be expressed in terms of the fundamental solution $F=F(x, t)$ by means of the variation of constants formula. Thus, for $t \geq 0$ we have

$$
\begin{aligned}
& H_{R}(x)=e^{-t} H_{R}(x)+\int_{\mathbb{R}^{N}} \omega(x-y, t) H_{R}(y) d y+\Lambda_{R}\left(\int_{0}^{t} e^{-(t-s)} d s\right) H_{R}(x) \\
& -\left(\int_{0}^{t} e^{-(t-s)} d s\right) \mathcal{X}_{B_{R}^{c}}(x)\left(J * H_{R}\right)(x)+\Lambda_{R} \int_{0}^{t} \int_{\mathbb{R}^{N}} \omega(x-y, t-s) H_{R}(y) d y d s \\
& -\int_{0}^{t} \int_{B_{R}^{c}} \omega(x-y, t-s)\left(J * H_{R}\right)(y) d y d s .
\end{aligned}
$$

For $x \in B_{R}$, there holds that $\mathcal{X}_{B_{R}^{c}}(x)=0$. Thus, we can rewrite (3.7) for $x \in B_{R}$ as,

$$
\begin{aligned}
\left(1-e^{-t}\right) & \left(1-\Lambda_{R}\right) H_{R}(x)= \\
= & \int_{\mathbb{R}^{N}} \omega(x-y, t) H_{R}(y) d y+\Lambda_{R} \int_{0}^{t} \int_{\mathbb{R}^{N}} \omega(x-y, t-s) H_{R}(y) d y d s \\
& -\int_{0}^{t} \int_{B_{R}^{c}} \omega(x-y, t-s)\left(J * H_{R}\right)(y) d y d s .
\end{aligned}
$$

Observe that we are free to select the parameter $t$ in expression (3.8).

Let us now rescale the identity (3.8). We have,

$$
\begin{aligned}
\left(1-e^{-t}\right) & \left(1-\Lambda_{R}\right) \widetilde{H}_{R}(x)= \\
= & \int_{\mathbb{R}^{N}} \omega(R x-y, t) H_{R}(y) d y+\Lambda_{R} \int_{0}^{t} \int_{\mathbb{R}^{N}} \omega(R x-y, t-s) H_{R}(y) d y d s \\
& \quad-\int_{0}^{t} \int_{B_{R}^{c}} \omega(R x-y, t-s)\left(J * H_{R}\right)(y) d y d s \\
:= & (i)+(i i)-(i i i) .
\end{aligned}
$$


In order to bound the derivatives of $(i),(i i)$ and (iii) we will choose the value $t=R^{2}$. First, let us estimate the derivative of $(i)$. By (3.5), since $0 \leq H_{R} \leq 1$, it follows that

$$
\begin{aligned}
\left|\nabla \int_{\mathbb{R}^{N}} \omega(R x-y, t) H_{R}(y) d y\right| & =R\left|\int_{\mathbb{R}^{N}} \nabla \omega(R x-y, t) H_{R}(y) d y\right| \\
& \leq R \int_{\mathbb{R}^{N}}|\nabla \omega(y, t)| d y \\
& \leq C R t^{-\frac{1}{2}}=C .
\end{aligned}
$$

Similarly, since $\Lambda_{R} \leq C R^{-2}$,

$$
\begin{aligned}
\Lambda_{R} \mid \nabla \int_{0}^{t} \int_{\mathbb{R}^{N}} & \omega(R x-y, t-s) H_{R}(y) d y d s \mid \\
& \leq \Lambda_{R} R \int_{0}^{t} \int_{\mathbb{R}^{N}}|\nabla \omega(y, t-s)| d y d s \\
\leq & C R^{-1} \int_{0}^{t}(t-s)^{-\frac{1}{2}} d s \\
& \leq C R^{-1} t^{\frac{1}{2}}=C .
\end{aligned}
$$

Now, by using the pointwise estimate (3.4), Corollary 3.5 and the fact that $\operatorname{supp}\left(J * H_{R}\right)=B_{R+1}$ we can bound the derivative of (iii) as

$$
\begin{aligned}
& \left|\nabla \int_{0}^{t} \int_{B_{R}^{c}} \omega(R x-y, t-s)\left(J * H_{R}\right)(y) d y d s\right|= \\
& \quad=R\left|\int_{0}^{t} \int_{B_{R}^{c}} \nabla \omega(R x-y, t-s)\left(J * H_{R}\right)(y) d y d s\right| \\
& \quad \leq C\left|\int_{0}^{t} \int_{R<|y|<R+1} \frac{t-s}{|R x-y|^{N+3}} d y d s\right| \\
& \quad \leq C t^{2} \int_{R<|y|<R+1} \frac{1}{|R x-y|^{N+3}} d y .
\end{aligned}
$$

Assume now, $|x| \leq r$ with $0<r<1$. Then, if $|y|>R$ we get that $|R x-y| \geq R(1-r)$ and then,

$$
\begin{aligned}
& \left|\nabla \int_{0}^{t} \int_{B_{R}^{c}} \omega(x-y, t-s)\left(J * H_{R}\right)(y) d y d s\right| \leq \\
& \quad \leq C R^{-N-3} t^{2} \frac{1}{(1-r)^{N+3}}|\{R<|y|<R+1\}| \\
& \quad \leq C_{r} R^{-4} t^{2}=C_{r} .
\end{aligned}
$$

Thus, since $\left(1-e^{-R^{2}}\right)\left(1-\Lambda_{R}\right) \geq \alpha_{0}>0$ for $R \geq R_{0}$, we conclude that for every $0<r<1$ there exists $C>0$ such that,

$$
\sup _{|x| \leq r}\left|\nabla \widetilde{H}_{R}(x)\right| \leq C
$$

if $R \geq R_{0}$.

We can apply Arzelà- Ascoli on every ball $B_{r}$ with $0<r<1$ to get, for every sequence $R_{n} \rightarrow \infty$ a subsequence $\widetilde{H}_{R_{n_{k}}}$ uniformly convergent in $B_{r}$. Then, a diagonal argument gives a subsequence uniformly convergent on every compact subset of $B_{1}$ to a function $H$. By the previous lemmas, we know that $H$ is a solution to

$$
-\Delta H=\lambda_{1} H \quad \text { in } \quad B_{1} .
$$


Moreover, $0 \leq H \leq 1$. Let us see that $H \in C\left(\bar{B}_{1}\right)$ with $H=0$ on $\partial B_{1}$. In fact, we show that the subsequence $\widetilde{H}_{R_{n_{k}}}$ converging to $H$ uniformly on compact subsets of $B_{1}$ is actually uniformly convergent in $B_{1}$. In fact, we use Lemma 3.4 to get for $\varepsilon>0$,

$$
\widetilde{H}_{R}(x) \leq C\left(\eta\left(\frac{|x|}{2}\right)-\eta\left(\frac{1}{2}\right)+\frac{C_{0}}{R}\right)<\frac{\varepsilon}{2}
$$

if $1-|x|<\delta_{0}$ and $R \geq R_{0}$.

On the other hand, for every $x \in B_{1}$, by taking limit as $k \rightarrow \infty$ we find that,

$$
H(x) \leq C\left(\eta\left(\frac{|x|}{2}\right)-\eta\left(\frac{1}{2}\right)\right)<\frac{\varepsilon}{2}
$$

if $1-|x|<\delta_{0}$.

Observe that, in particular, $H \in C\left(\bar{B}_{1}\right)$ with $H=0$ on $\partial B_{1}$.

Then,

$$
\left|\widetilde{H}_{R_{n_{k}}}(x)-H(x)\right| \leq \varepsilon \quad \text { if } \quad|x|>1-\delta_{0}, \quad k \geq k_{0} .
$$

On the other hand, due to the uniform convergence of $\widetilde{H}_{R_{n_{k}}}$ to $H$ in $\bar{B}_{1-\delta_{0}}$,

$$
\left|\widetilde{H}_{R_{n_{k}}}(x)-H(x)\right| \leq \varepsilon \quad \text { if } \quad|x| \leq 1-\delta_{0}, \quad k \geq k_{1} .
$$

So that, the convergence is uniform in $B_{1}, H \in C\left(\bar{B}_{1}\right)$ with $H=0$ on $\partial B_{1}$. So that, $H=h_{1}$ is independent of the subsequence and the theorem is proved.

\section{Back to the evolutionary problem. Construction of a Barrier.}

In this section we construct a barrier for the nonlocal problem which is similar to the one constructed in [13] for the laplacian. This barrier is a function of separated variables involving the eigenfunctions $H_{R}$ studied in Section 3.

In order to be able to further analyze our solution $u$, we state a result that is needed because of the lack of a regularizing effect of the nonlocal diffusion equation.

Lemma 4.1. Let $0 \leq u_{0} \in L^{\infty}, u_{0} \not \equiv 0$. Then, for every $R>0, t>0$,

$$
\inf _{x \in B_{R}} u(x, t)>0 .
$$

Proof. We recall some results that can be found, for instance, in [17]. First, $u \in L^{\infty}$ and bounded by $\left\|u_{0}\right\|_{\infty}$. Moreover, $u \geq 0$ since $v \equiv 0$ is a solution to the equation and a comparison principle for bounded solutions holds (see, for instance [15]).

Moreover, $u(x, t)>0$ for every $x \in \mathbb{R}^{N}, t>0$. In fact, let $A \geq 1+\left\|u_{0}\right\|_{\infty}^{p-1}$. Then, since $0 \leq u \leq\left\|u_{0}\right\|_{\infty}$,

$$
u_{t}+A u \geq u_{t}+u+u^{p}=J * u \text {. }
$$

Thus,

$$
u(x, t) \geq e^{-A t} u_{0}(x)+\int_{0}^{t} e^{-A(t-s)}(J * u(\cdot, s))(x) d x
$$

so that, if $u(x, t)=0$ for some $x \in \mathbb{R}^{N}, t>0$ there holds,

$$
0 \geq \int_{0}^{t} e^{-A(t-s)}(J * u(\cdot, s))(x) d x \geq 0 .
$$

We deduce that $u(y, s)=0$ in $B_{1}(x) \times(0, t)$ and, since $\mathbb{R}^{N}$ is connected, a continuation argument gives that $u=0$ in $\mathbb{R}^{N} \times(0, t)$. But, by $(4.2)$,

and $u_{0} \not \equiv 0$.

$$
u(x, t) \geq e^{-A t} u_{0}(x)
$$

Therefore, $u(x, t)>0$ in $\mathbb{R}^{N} \times(0, \infty)$. 
Let us now prove (4.1). In not, there exists a sequence $\left\{x_{n}\right\} \subset B_{R}$ such that $u\left(x_{n}, t\right) \rightarrow 0$. Without loss of generality we may assume that $x_{n} \rightarrow \bar{x} \in \bar{B}_{R}$. Going back to (4.2) and using that $J * u(\cdot, s)$ is a continuous function in $\mathbb{R}^{N}$ we get

$$
0 \leftarrow u\left(x_{n}, t\right) \geq \int_{0}^{t} e^{-A(t-s)}(J * u(\cdot, s))\left(x_{n}\right) d x \rightarrow \int_{0}^{t} e^{-A(t-s)}(J * u(\cdot, s))(\bar{x}) d x .
$$

We deduce that $u=0$ in $B_{1}(\bar{x}) \times(0, t)$, a contradiction.

Now, we construct the barrier.

Lemma 4.2. Let $\Lambda_{R}$ be the principal eigenvalue of (2.3) in the ball $B_{R}$ and $H_{R}$ the positive eigenfunction with the normalization $\left\|H_{R}\right\|_{L^{\infty}\left(B_{R}\right)}=1$. Assume $0 \leq u_{0} \in L^{\infty}\left(\mathbb{R}^{N}\right)$ and let $u$ be the unique bounded solution of (1.1). Then, the following inequality holds in $B_{R} \times(0,+\infty)$ :

$$
u(x, t) \geq \psi_{R}(t) H_{R}(x),
$$

where $\psi_{R}$ is the solution to

$$
\left\{\begin{array}{l}
\frac{d}{d t} \psi_{R}+\Lambda_{R} \psi_{R}+\psi_{R}^{p}=0 \\
\psi_{R}(0)=c=\inf _{x \in B_{R}} \frac{u_{0}(x)}{H_{R}(x)} .
\end{array}\right.
$$

Proof. We set $w(x, t)=\psi_{R}(t) H_{R}(x)$. Then, for $x \in B_{R}$,

$$
\begin{aligned}
w_{t}-\mathcal{L} w+w^{p} & =H_{R} \frac{d}{d t} \psi_{R}-\psi_{R} \mathcal{L} H_{R}+\psi_{R}^{p} H_{R}^{p} \\
& =H_{R} \frac{d}{d t} \psi_{R}+\psi_{R} \Lambda_{R} H_{R}+\psi_{R}^{p} H_{R}^{p} \\
& =H_{R}\left(\frac{d}{d t} \psi_{R}+\Lambda_{R} \psi_{R}+\psi_{R}^{p}\right)+H_{R} \psi_{R}\left(\left(H_{R} \psi_{R}\right)^{p-1}-\psi_{R}^{p-1}\right) .
\end{aligned}
$$

Since $\psi_{R}$ satisfies (4.4), $0 \leq H_{R} \leq 1$ and $p \geq 1$ we deduce that,

$$
w_{t}-\mathcal{L} w+w^{p} \leq 0 \quad \text { for } x \in B_{R} .
$$

As $w(x, 0)=\psi_{R}(0) H_{R}(x) \leq u_{0}(x)$ and $w(x, t)=0$ in $B_{R}^{c} \times(0, \infty)$, we deduce by the comparison principle for sub- and super-solutions on bounded sets that,

$$
w(x, t) \leq u(x, t)
$$

Remark 4.3. The function $\psi$ can be computed explicitly (see [13]). In fact, if $c>0$,

$$
\psi_{R}(t)=\left(\frac{\Lambda_{R}}{\left(1+c^{1-p} \Lambda_{R}\right) e^{\Lambda_{R}(p-1) t}-1}\right)^{\frac{1}{p-1}} .
$$

The following technical lemma was proved in [13]. This result will be used later on in Section 5 in order to obtain the region where we can identify the asymptotic behavior of $u$.

Lemma 4.4 (Gmira and Veron, Lemma 2.2, [13]). Set $\varphi: \mathbb{R}^{+} \rightarrow \mathbb{R}^{+}$such that

$$
\lim _{y \rightarrow \infty} y^{2} \varphi(y)=\infty
$$

Then, there exists a nondecreasing function $R$ from $\mathbb{R}^{+}$into $\mathbb{R}^{+}$such that

$$
\lim _{y \rightarrow \infty} \frac{y}{R^{2}(y)}=0, \quad \lim _{y \rightarrow \infty} y \varphi(R(y))=\infty .
$$

Remark 4.5. In [13] the function $\varphi$ was assumed continuous. But it is easy to see that this assumption is not needed. 
Now, we prove a key lemma. Once again the goal is to establish a lower bound for $u(\cdot, t)$ by constructing an appropriate auxiliary function $\varphi(R)$. This function will be used as an initial condition for the function $\psi_{R}$ from Lemma 4.2 in the proof of Theorem 5.1.

Proposition 4.6. Suppose $0 \leq u_{0} \in L^{\infty}\left(\mathbb{R}^{N}\right)$ is such that

$$
|x|^{\frac{2}{p-1}} u_{0}(x) \rightarrow \infty \quad \text { as } \quad|x| \rightarrow \infty
$$

and let $u$ be the bounded solution to (1.1). Then, for any $t>0$ the following equivalent properties hold:

(i) $\lim _{|x| \rightarrow \infty}|x|^{\frac{2}{p-1}} u(x, t)=\infty$.

(ii) $\lim _{R \rightarrow \infty} R^{\frac{2}{p-1}} \inf _{|x| \leq R} u(x, t)=\infty$.

(iii) There exists a positive, non-increasing, real-valued function $\varphi$ such that

$$
\lim _{r \rightarrow \infty} r^{\frac{2}{p-1}} \varphi(r)=\infty
$$

and,

$$
u(x, t) \geq \varphi(R) H_{R}(x), \quad \forall x \in B_{R} .
$$

Proof. By (4.2), for every $t>0$,

$$
|x|^{\frac{2}{p-1}} u(x, t) \geq e^{-A t}|x|^{\frac{2}{p-1}} u_{0}(x) \rightarrow \infty \quad \text { as } \quad|x| \rightarrow \infty .
$$

Thus, (i) holds.

Let us see that (i) $\Rightarrow$ (ii).

If not, there exist $R_{n} \rightarrow \infty$ and a constant $C>0$ such that

$$
R_{n}^{\frac{2}{p-1}} \inf _{B_{R_{n}}} u(\cdot, t) \leq C .
$$

This in turn implies that there exists $x_{n} \in B_{R_{n}}$ such that

$$
R_{n}^{\frac{2}{p-1}} u\left(x_{n}, t\right) \leq 2 C .
$$
4.1 ,

If there exist $R_{0}>0$ and a subsequence $R_{n_{k}}$ such that $\left\{x_{n_{k}}\right\} \subset B_{R_{0}}$ we would have, by (4.9) and Lemma

$$
2 C \geq R_{n_{k}}^{\frac{2}{p-1}} u\left(x_{n_{k}}, t\right) \geq R_{n_{k}}^{\frac{2}{p-1}} \inf _{B_{R_{0}}} u(\cdot, t) \rightarrow \infty \quad \text { as } \quad k \rightarrow \infty
$$

which is a contradiction. Therefore, $\left|x_{n}\right| \rightarrow \infty$ as $n \rightarrow \infty$. But then, since $x_{n} \in B_{R_{n}}$, by $(i)$,

$$
2 C \geq R_{n}^{\frac{2}{p-1}} u\left(x_{n}, t\right) \geq\left|x_{n}\right|^{\frac{2}{p-1}} u\left(x_{n}, t\right) \rightarrow \infty \quad \text { as } \quad n \rightarrow \infty
$$

which again is a contradiction. So, (ii) holds.

(ii) $\Rightarrow$ (iii). We define for $R>0$

$$
\varphi(R)=\inf _{|x| \leq R} \frac{u(x, t)}{H_{R}(x)},
$$

where $H_{R}$ is the positive eigenfunction of (2.3) with $\left\|H_{R}\right\|_{\infty}=1$. As $u(x, t) / H_{R} \geq u(x, t)$ in $B_{R}$, there holds that $\varphi(R)$ is positive.

From (4.10) we have in $B_{R}$,

and, as $0 \leq H_{R}(x) \leq 1$,

$$
u(x, t) \geq \varphi(R) H_{R}(x)
$$

$$
R^{\frac{2}{p-1}} \varphi(R) \geq R^{\frac{2}{p-1}} \inf _{|x| \leq R} u(x, t) \rightarrow \infty \quad \text { as } \quad R \rightarrow \infty,
$$

by (ii). So that, (iii) holds.

(iii) $\Rightarrow$ (ii). In fact, by Theorem 3.1 we know that

$$
\widetilde{H}_{R}(x) \rightarrow h_{1} \text { uniformly in } B_{1 / 2} .
$$


Since $h_{1}(x) \geq \beta>0$ in $B_{1 / 2}$, there holds that

$$
\widetilde{H}_{R}(x) \geq \frac{\beta}{2} \quad \text { in } \quad B_{1 / 2}
$$

if $R \geq R_{0}$.

This is,

$$
H_{R}(x) \geq \frac{\beta}{2} \quad \text { in } \quad B_{R / 2}
$$

if $R \geq R_{0}$. Hence,

$$
u(x, t) \geq \varphi(R) H_{R}(x) \geq \frac{\beta}{2} \varphi(R) \quad \text { in } \quad B_{R / 2}
$$

if $R \geq R_{0}$.

Multiplying by $R^{\frac{2}{p-1}}$, taking infimum over $B_{R / 2}$ and letting $R \rightarrow \infty$ gives (ii).

(ii) trivially implies (i).

Remark 4.7. Observe that the function $\varphi(R)$ depends on $t>0$.

\section{MAin Result}

In this section we prove our main result. This is, we obtain the large time behavior of $u$ in the subcritical case $1<p<1+2 / \alpha$.

Theorem 5.1. Suppose $0 \leq u_{0} \in L^{\infty}\left(\mathbb{R}^{N}\right)$ satisfies (4.7). Let $u(x, t)$ be the bounded solution of (1.1). Then,

$$
\lim _{t \rightarrow \infty} t^{\frac{1}{p-1}} u(x, t)=\left(\frac{1}{p-1}\right)^{\frac{1}{p-1}},
$$

uniformly on the sets

$$
E_{k}=\left\{x \in \mathbb{R}^{N}:|x| \leq k \sqrt{t}\right\}
$$

where $k$ is an arbitrary constant.

Proof. From Proposition 4.6, by considering $u(x, t)$ for $t \geq t_{0}>0$ we deduce that there is no loss of generality in assuming that there exists a nondecreasing function $\varphi: \mathbb{R}^{+} \rightarrow \mathbb{R}^{+}$satisfying (4.8) such that,

$$
u_{0}(x) \geq \varphi(R) H_{R}(x) \quad \forall x \in B_{R} .
$$

From Lemma 4.2 we have that $u(x, t) \geq H_{R}(x) \psi_{R}(t)$ in $B_{R} \times \mathbb{R}^{+}$, where $\psi_{R}$ is the solution of

$$
\left\{\begin{array}{l}
\frac{d}{d t} \psi_{R}+\Lambda_{R} \psi_{R}+\psi_{R}^{p}=0 \quad \text { in }(0, \infty) \\
\psi_{R}(0)=\varphi(R) .
\end{array}\right.
$$

By (2.4) the principal eigenvalue of (2.3) we can written in the form

$$
\Lambda_{R}=C_{R} \frac{\lambda_{1}}{R^{2}} \quad \text { as } \quad R \rightarrow+\infty,
$$

where $\lambda_{1}$ is the principal eigenvalue of $(2.1)$ and $C_{R} \rightarrow A(J)$ as $R \rightarrow \infty$ with $A(J)$ given by (2.5). By using (5.2) and (4.5) we have

$$
\begin{aligned}
t^{\frac{1}{p-1}} \psi_{R}(t) & =t^{\frac{1}{p-1}}\left(\frac{\Lambda_{R}}{\left(1+\varphi^{1-p}(R) \Lambda_{R}\right) e^{\Lambda_{R}(p-1) t}-1}\right)^{\frac{1}{p-1}} \\
& =\frac{\left(C_{R} t \lambda_{1} R^{-2}\right)^{\frac{1}{p-1}} e^{-C_{R} \lambda_{1} t R^{-2}}}{\left(1+C_{R} \lambda_{1} \varphi^{1-p}(R) R^{-2}-e^{-C_{R} \lambda_{1}(p-1) t R^{-2}}\right)^{\frac{1}{p-1}}} .
\end{aligned}
$$


By using the Taylor expansion for $e^{-C_{R} \lambda_{1}(p-1) t R^{-2}}$ at the origin we get,

$$
t^{\frac{1}{p-1}} \psi_{R}(t)=\left(\frac{\frac{C_{R} t \lambda_{1}}{R^{2}}}{\frac{C_{R} \lambda_{1}}{R^{2} \varphi^{p-1}(R)}+\frac{C_{R} \lambda_{1}(p-1) t}{R^{2}}+O\left(R^{-4} t^{2}\right)}\right)^{\frac{1}{p-1}} e^{-C_{R} \lambda_{1} t R^{-2}} .
$$

Now, as $\lim _{R \rightarrow \infty} R^{2} \varphi^{p-1}(R)=\infty$ we deduce from Lemma 4.4 that there exists a nondecreasing function $t \mapsto R(t)$ such that

$$
\lim _{t \rightarrow \infty} \frac{t}{R^{2}(t)}=0 \quad \text { and } \quad \lim _{t \rightarrow \infty} t \varphi^{p-1}(R(t))=\infty .
$$

Replacing $R$ by $R(t)$ in (5.3) and using (5.4) yields

$$
\lim _{t \rightarrow \infty} t^{\frac{1}{p-1}} \psi_{R(t)}(t)=\left(\frac{1}{p-1}\right)^{\frac{1}{p-1}}
$$

If we consider $x$ such that $\frac{|x|}{\sqrt{t}} \leq k$ for some constant $k$, we have

$$
\lim _{t \rightarrow \infty} \frac{|x|}{R(t)}=\lim _{t \rightarrow \infty} \frac{|x|}{\sqrt{t}} \frac{\sqrt{t}}{R(t)}=0
$$

Since $w(x, t)=\left(\frac{1}{(p-1) t}\right)^{\frac{1}{p-1}}$ is a supersolution for $t>0$, and $u$ is bounded there holds that,

$$
\left(\frac{1}{p-1}\right)^{\frac{1}{p-1}} \geq t^{\frac{1}{p-1}} u(x, t) \geq t^{\frac{1}{p-1}} \psi_{R(t)}(t) H_{R(t)}(x) .
$$

Now, let us prove that

$$
\lim _{t \rightarrow \infty} H_{R(t)}(x)=1
$$

uniformly on $|x| \leq k \sqrt{t}$ for all $k>0$. In fact, from the uniform convergence on $B_{1}$ obtained in Theorem 3.1, for every $\varepsilon>0$ there exists $t_{1}>0$ such that,

$$
\left|\widetilde{H}_{R(t)}(y)-h_{1}(y)\right|<\varepsilon \quad \text { if } \quad|y| \leq 1 \quad \text { and } \quad t \geq t_{1} .
$$

On the other hand, if $|x| \leq k \sqrt{t}$ and we put $x=R(t) y$, we get that

$$
|y| \leq \frac{k \sqrt{t}}{R(t)} \leq 1 \quad \text { if } t \geq t_{2}
$$

and consequently, if $|x| \leq k \sqrt{t}$

$$
\left|H_{R(t)}(x)-h_{1}\left(\frac{x}{R(t)}\right)\right|=\left|\widetilde{H}_{R(t)}(y)-h_{1}(y)\right|<\varepsilon \quad \text { if } \quad t \geq \max \left\{t_{1}, t_{2}\right\} .
$$

From the continuity of $h_{1}$ it follows that,

$$
\left|h_{1}\left(\frac{x}{R(t)}\right)-h_{1}(0)\right| \leq \varepsilon \quad \text { if } \quad|x| \leq k \sqrt{t} \quad \text { and } \quad t \geq t_{3} .
$$

Hence, from (5.8) and (5.9) we obtain that,

$$
\left|H_{R(t)}(x)-h_{1}(0)\right|<2 \varepsilon \quad \text { if } \quad|x| \leq k \sqrt{t} \quad \text { and } \quad t \geq\left\{t_{1}, t_{2}, t_{3}\right\} .
$$

Since $h_{1}(0)=1,(5.7)$ follows.

Taking limit as $t \rightarrow \infty$ in (5.6) and using (5.7) we obtain that

$$
\lim _{t \rightarrow \infty} t^{\frac{1}{p-1}} u(x, t)=\left(\frac{1}{p-1}\right)^{\frac{1}{p-1}}
$$

uniformly on $E_{k}=\left\{x \in \mathbb{R}^{N}:|x| \leq k \sqrt{t}\right\}$ and the proof is finished. 


\section{REFERENCES}

[1] F. Andreu, J. Mazón, J. D. Rossi, J. Toledo, A nonlocal p-Laplacian evolution equation with nonhomogeneous Dirichlet boundary conditions, SIAM J. Math. Anal. 40(5), 2008/09, 1815-1851.

[2] P. Bates, A. Chmaj, An integrodifferential model for phase transitions: Stationary solutions in higher dimensions, J. Statistical Phys. 95, 1999, 1119-1139.

[3] P. Bates, A. Chmaj, A discrete convolution model for phase transitions, Arch. Rat. Mech. Anal. 150, 1999, $281-305$.

[4] P. Bates, P. Fife, X. Ren, X. Wang, Travelling waves in a convolution model for phase transitions, Arch. Rat. Mech. Anal. 138, 1997, 105-136.

[5] P. Bates, G. Zhao Existence, Uniqueness and Stability of the Stationary Solution to a Nonlocal Evolution Equation Arising in Population Dispersal, J. Math. Anal. Appl., 332, 2007, 428-440.

[6] C. Carrillo, P. Fife, Spatial effects in discrete generation population models, J. Math. Biol. 50(2), 2005, 161-188.

[7] M. Chaves, E. Chasseigne, J. D. Rossi, Asymptotic behavior for nonlocal diffusion equations, Adv. Differential Equations, 2, 2006, 271-291.

[8] C. Cortazar, M. Elgueta, F. Quiros, N. Wolanski, Asymptotic Behavior for a Nonlocal Diffusion Equation in Domains with Holes, Arch. Rational Mech. Anal. 205, 2012, 673-697.

[9] P. Fife, Some nonclassical trends in parabolic and parabolic-like evolutions, Trends in nonlinear analysis, 153-191, Springer, Berlin, 2003.

[10] J. García-Melián, F. Quirós, Fujita exponents for evolution problems with nonlocal diffusion, J. Evol. Equ. 10(1), 2010, $147-161$.

[11] J. García-Melián, J. D. Rossi, On the principal eigenvalue of some nonlocal diffusion problems, J. Differential Equations 246(1), 2009, 21-38.

[12] G. Gilboa, S. Osher, Nonlocal operators with application to image processing, Multiscale Model. Simul., 7(3), 2008, 10051028.

[13] A. Gmira, L. Veron, Large Time Behaviour of the Solutions of a Semilinear Parabolic Equation in $R^{N}$, Jour. Diff. Eqs. 53, 1984, 258-276.

[14] Ignat, J. D. Rossi, Refined asymptotic expansions for nonlocal diffusion equations, J. Evolution Equations. 8, 2008, 617629.

[15] C. Lederman, N. Wolanski, Singular perturbation in a nonlocal diffusion model, Communications in PDE 31(2), 2006, $195-241$.

[16] A. Pazoto, J. D. Rossi, Asymptotic behavior for a semilinear nonlocal equation. Asymptotic Analysis. 52(1-2), 2007, $143-155$.

[17] J. Terra, N. Wolanski, Asymptotic behavior for a nonlocal diffusion equation with absorption and nonintegrable initial data. The supercritical case, Proc. Amer. Math. Soc., 139 (4), 2011, 1421-1432.

[18] J. Terra, N. Wolanski, Large time behavior for a nonlocal diffusion equation with absorption and bounded initial data, Discrete Cont. Dyn. Syst. A, 31 (2011) 2, 581-605.

[19] L. Zhang, Existence, uniqueness and exponential stability of traveling wave solutions of some integral differential equations arising from neuronal networks, J. Differential Equations 197(1), 2004, 162-196.

Ariel SALORT

IMAS-CONICET AND

Departamento de Matemática, FCEyN-UBA

(1428) Buenos Aires, Argentina.

E-mail address: asalort@dm.uba.ar

JOANA TERRA

IMAS-CONICET AND

Departamento de Matemática, FCEyN-UBA

(1428) Buenos Aires, Argentina.

E-mail address: jterra@dm.uba.ar

NoEmi Wolanski

IMAS-CONICET AND

Departamento de Matemática, FCEyN-UBA

(1428) Buenos Aires, Argentina.

E-mail address: wolanski@dm.uba.ar 